\title{
Soft X-Ray Microscopy for Probing of Topical Tacrolimus Delivery via Micelles
}

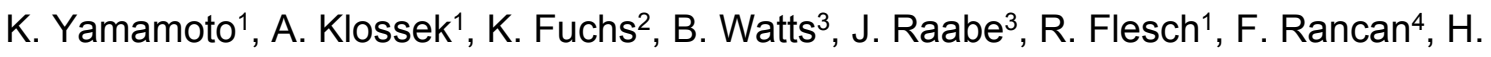

Pischon $^{5}$, M. Radbruch ${ }^{5}$, A.D. Gruber ${ }^{5}$, L. Mundhenk ${ }^{5}$, A. Vogt ${ }^{4}$, U. Blume-Peytavi ${ }^{4}$, P. Schrade $^{6}$, S. Bachmann ${ }^{6}$, R. Gurny ${ }^{2}$, and E. Rühl ${ }^{1}$

${ }^{1}$ Physikalische Chemie, Freie Universität Berlin, Takustr. 3, 14195 Berlin, Germany ${ }^{2}$ Apidel SA, c/o The Business Harbour, 29 Quai du Mont Blanc, 1201 Geneva, Switzerland ${ }^{3}$ Swiss Light Source, Paul Scherrer Institut, Forschungsstraße 111, 5232 Villigen PSI, Switzerland

${ }^{4}$ Clinical Research Center for Hair and Skin Science, Department of Dermatology and Allergy, Charité - Universitätsmedizin Berlin, corporate member of Freie Universität Berlin, Humboldt-Universität zu Berlin, and Berlin Institute of Health, 10117 Berlin, Germany ${ }^{5}$ Institute of Veterinary Pathology, Freie Universität Berlin, Robert-von-Ostertag-Str. 15, 14163 Berlin, Germany

${ }^{6}$ Abteilung für Elektronenmikroskopie at CVK, 13353 Berlin Germany

\begin{abstract}
The penetration of topically applied tacrolimus formulated in micelles into murine skin is reported, measured by X-ray microscopy. Tacrolimus and micelles are probed for the first time by this high spatial resolution technique by element-selective excitation in the $C 1 \mathrm{~s}$ - and $\mathrm{O}$ 1s-regimes. This method allows selective detection of the distribution and penetration depth of drugs and carrier molecules into biologic tissues. It is observed that small, but distinct quantities of the drug and micelles, acting as a drug carrier, penetrate the stratum corneum. A comparison is made with the paraffin-based commercial tacrolimus ointment Protopic ${ }^{\circledR}$, where local drug concentrations show to be low. A slight increase in local drug concentration in the stratum corneum is observed, if tacrolimus is formulated in micelles, as compared to Protopic ${ }^{\circledR}$. This underscores the importance of the drug formulations for effective drug delivery. Time-resolved penetration shows presence of drug in the stratum corneum $100 \mathrm{~min}$ after formulation application, with penetration to deeper skin layers at 1000 min. High resolution micrographs give indications for a penetration pathway along the lipid membranes between corneocytes, but also suggest that the compound may penetrate corneocytes.
\end{abstract}

This document is the accepted manuscript version of the following article: Yamamoto, K., Klossek, A., Fuchs, K., Watts, B., Raabe, J., Flesch, R., ... Rüh1, E. (2019). Soft X-ray microscopy for probing of topical tacrolimus delivery via micelles. European Journal of Pharmaceutics and Biopharmaceutics, 139, 68-75. https:// doi.org/10.1016/j.ejpb.2019.03.006 


\section{Introduction}

The sensitive detection of topically applied drugs within skin layers is an important issue, which has been addressed in various studies [1, 2]. Some drugs easily penetrate into viable skin layers or even beyond via transdermal penetration, whereas others do not penetrate and remain essentially on the skin surface. This has been empirically determined for lipophilic drugs by the 500 Da rule, which implies that above this molecular mass efficient penetration is substantially impeded [3]. The essential barrier for this process is the stratum corneum. It was also shown that in inflammatory skin diseases, such as atopic dermatitis, the barrier function is decreased and the cutoff limit of penetrating substances is shifted to higher molecular weights [3]. Psoriasis-like lesions, epidermal hyperplasia, and hyperkeratosis may also affect the penetration of anti-inflammatory drugs [4].

Probing drugs encapsulated in nanoscopic particles after topical application on skin requires specific detection techniques, which have been reviewed recently [5]. Preferably, label-free approaches are required, by exploiting the inherent properties of the drugs and drug carriers. Several experimental approaches have been employed for label-free detection, which includes Raman-based approaches [6], atomic force microscopy [7], including tip-enhanced Raman scattering [8], photoacoustic nanoscopy [9], photothermal expansion [10], and X-ray microscopy [11].

Recently, the penetration of the lipophilic drug dexamethasone by scanning X-ray microscopy was reported [11-13]. These studies yielded quantitatively systematic findings at different penetration times, so that the amount of drug in different skin layers was determined. The central advantage of X-ray microscopy is that the results can be quantified due to Beer-Lambert law, In $\left(I / I_{0}\right)=-\sigma c \cdot d$, where $I$ is the transmitted radiation, $I_{0}$ is the incident radiation, $\sigma$ is the absorption cross section measured in Mbarn (1 Mbarn corresponds to $10^{-18} \mathrm{~cm}^{2}$ ), $c$ is the local concentration, and $d$ is the thickness of the sample that is penetrated by soft X-rays. The quantity $\ln \left(I / I_{0}\right)$ is also called differential absorption, probing the relative change of the transmitted radiation through the sample. Most of dexamethasone is found in the stratum corneum and there specifically in the lipid lamellae between the corneocytes, as follows from high resolution studies [12]. A characteristic minimum in local drug concentration is found near the stratum granulosum, indicating that tight junctions are a further barrier for drug penetration [14], as was analyzed in detail by a transport model [13]. Beyond the viable skin the drug is only found in the viable epidermis, hinting that the basal membrane is another barrier impeding drug permeation to the dermis. In addition, stratum corneum removal by repeated tape stripping yielded an enhanced drug penetration behavior, which is due to the thinned stratum corneum. As a result, the drug 
penetrated in shorter time into deeper skin layers [12]. In addition, the drug transport was modified by core-multishell nanocarriers, which transport dexamethasone more slowly through the stratum corneum and release the drug in the lower part of this top skin layer [15]. As a result, less drug is contained in the stratum corneum and the drug is transported in larger local concentrations into the viable epidermis. Interestingly, the nanocarriers remained in the stratum corneum and were not able to cross the tight junction zone. Similar findings were reported from labeled nanocarriers, which were probed by fluorescence microscopy [16]. Even in inflamed skin of murine models of psoriasis or atopic dermatitis the nanocarriers remained in the stratum corneum $[17,18]$.

These previous findings provide the motivation for the herein presented work. We have modified the experimental parameters as follows: instead of dexamethasone we chose the higher molecular weight drug tacrolimus $\left(\mathrm{C}_{44} \mathrm{H}_{69} \mathrm{NO}_{12} ; \mathrm{m}=804.02 \mathrm{~g} / \mathrm{mol}\right.$, see Scheme 1(a), which is used in atopic dermatitis [19] and psoriasis [20] treatment. The compound is known to be difficult to formulate and penetrates only in small quantities into human skin [21]. The dermal penetration of tacrolimus has been investigated before, where it was shown that the formulation changes the drug penetration profile at low spatial resolution of $20 \mu \mathrm{m}$, as assessed by analytical approaches, such as ultrahigh pressure liquid chromatography with tandem mass spectrometry detection [22]. The result of this study was that a higher amount of tacrolimus was found mainly in the stratum corneum and up to the superficial dermis, if micelles containing the drug were used, as compared to the commercial Protopic ${ }^{\circledR}$ formulation. Subsequent work on skin delivery of a tacrolimus composite containing hydrogel in an imiquimod-induced psoriasis mouse model yielded a twice higher drug concentration than the commercial formulation Protopic ${ }^{\circledR}$, which was also used as a benchmark [21]. Alternatively, transferosomes have been employed for efficient delivery of tacrolimus [23]. Most recently, various delivery strategies for tacrolimus have been reviewed [24]. In this work we exposed inflamed murine skin to a formulation containing tacrolimus embedded in micelles for determining the penetration of these species (cf. Scheme 1). This includes the aspect of enhanced drug penetration due to skin inflammation and the novel formulation of this strongly lipophilic drug $(\log P=3.96 \pm 0.83$ [25]). It is known that imiquimod-induced psoriasis-like skin lesions lead to enhanced skin permeability and drug penetration for both hydrophilic and hydrophobic drugs [4]. This also includes tacrolimus, which showed an about ten-fold increased flux in psoriatic skin compared to murine healthy skin. 
(b)

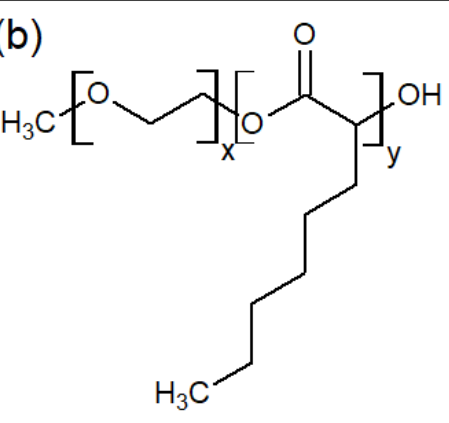

(c)

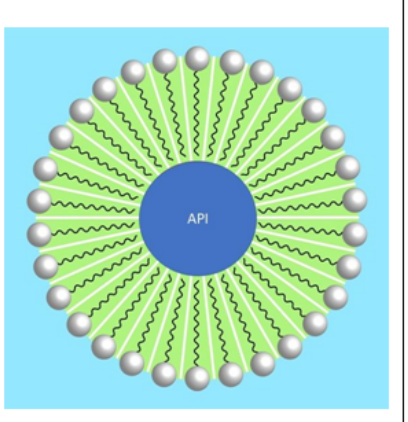

Scheme 1: (a) Structure of tacrolimus (taken from Ref. [26]); (b) structure of the polymer forming micelles for transporting tacrolimus ( $x \simeq 40-50$ units, $y \simeq 20-30$ units)); (c) structure of a micelle incorporating the active pharmaceutical ingredient (API) tacrolimus (hydrodynamic diameter $\sim 30 \mathrm{~nm}$ ), where hydrophobic polymer chains point toward the inside and hydrophilic polymer chains toward the outside of the micellar corona.

\section{Materials and Methods}

Tacrolimus (TAC) $>99 \%$ (cf. Scheme 1(a)) was purchased from LC Laboratories (Woburn, MA, USA). Sodium citrate dihydrate and anhydrous citric acid $\mathrm{Ph}$. Eur. grade were purchased from Haenseler (Herisau, Switzerland). Methoxy poly(ethylene glycol) hexylsubstituted poly(lactic acid) (mPEGhexPLA or methoxy PEG poly(caprylic acid)) (molecular weight: $5.5 \mathrm{kDa}$ ) was supplied by Apidel SA, Geneva, Switzerland. Acetonitrile HPLC grade (HPLC: high pressure liquid chromatography), water HPLC grade (Biosolve ${ }^{\circledR}$ ), and sterile water (Corning ${ }^{\circledR}$ ) were obtained from Brunschwig (Basel, Switzerland). Acetone Ph. Eur., trifluoroacetic acid (TFA, HPLC grade) were purchased from Sigma, Buchs, Switzerland.

Placebo and $0.7 \%$ TAC-mPEG hexyl-substituted poly(lactic acid) micellar formulations (cf. Scheme 1(b), (c)) were prepared in citrate buffer at a $5 \mathrm{~mL}$ batch scale. Briefly, tacrolimus and polymers were dissolved in a 1:15 ratio (tacrolimus: polymer, w/w) in $2 \mathrm{~mL}$ of acetone. This organic phase was added dropwise $(6 \mathrm{~mL} / \mathrm{h})$ to the citrate buffer $(20 \mathrm{mM}, \mathrm{pH} 5.5)$ under sonication (amplitude 20\%, SD450, Branson, USA). Acetone was evaporated under reduced pressure $\left(60{ }^{\circ} \mathrm{C}, 180 \mathrm{mbar}\right.$, Rotavapor R210, Buechi, Switzerland) and the final volume of the formulation was controlled to ensure the correct tacrolimus dose. Placebo mPEG hexylsubstituted poly(lactic acid) micellar formulations were prepared accordingly, but without tacrolimus. Finally, the micelles were filtered under a laminar flow hood through $0.22 \mu \mathrm{m}$ polyvinylidene fluoride (PVDF) filters into sterilized vials and kept at $5{ }^{\circ} \mathrm{C}$.

The formulations were characterized in terms of drug content, micelle size, and $\mathrm{pH}$. The tacrolimus content was quantified by high pressure liquid chromatography (HPLC, Agilent 1100, Basel, Switzerland) with a Kinetex Phenomenex ${ }^{\circledR}$ XB-C18 column (75 × 3 mm I.D., 2.6 $\mu \mathrm{m})$ heated to $50{ }^{\circ} \mathrm{C}$. The analysis was carried out in gradient mode at a flow rate of 1 
$\mathrm{mL} / \mathrm{min}$ over $7 \mathrm{~min}$. The mobile phase consisted of a mixture of water with $0.1 \%(\mathrm{v} / \mathrm{v})$ TFA (A) and acetonitrile with $0.1 \%(\mathrm{v} / \mathrm{v})$ TFA (B). A linear gradient from $45 \%$ to $55 \%$ B was applied for $4 \mathrm{~min}$, followed by $2 \mathrm{~min}$ at $55 \% \mathrm{~B}$ and an equilibration step down to $45 \%$ B in $1 \mathrm{~min}$. The injection volume was set to $20 \mu \mathrm{L}$ and UV detection was performed at $205 \mathrm{~nm}$. The particle size was characterized by dynamic light scattering using back scattering light $\left(173^{\circ}\right)$ with a Zetasizer Nano-ZS (Malvern Instruments, UK). Micelle size and distribution were measured in triplicates and expressed as number weighted $\left(d_{n}\right)$ and intensity weighted (Z-average, $\left.Z_{a v}\right)$ hydrodynamic diameters and polydispersity index (PDI). The $Z_{a v}$ of both the tacrolimusloaded and neat micelles was around $30 \mathrm{~nm}$ and the target load with tacrolimus was $0.7 \%$.

Murine skin with imiquimod-induced psoriasis-like dermatitis gathered in an in vivo experiment was used for these ex vivo experiments [17]. The frozen skin samples were stored at $-80^{\circ} \mathrm{C}$ and were thawed for these studies and warmed to $37^{\circ} \mathrm{C}$ before incubation. Neat and tacrolimus-loaded micelles and $0.1 \%$ Protopic $^{\circledR}$ were exposed to murine skin so that all samples were treated topically with a drug concentration of $437 \mu \mathrm{g} / \mathrm{cm}^{2}$ at $37^{\circ} \mathrm{C}$, i.e. the following samples were prepared: (i) reference skin treated for $100 \mathrm{~min}$ with physiological $\mathrm{NaCl}$ solution; (ii) $0.1 \%$ Protopic $^{\circledR}$ (100 min penetration time); (iii) neat micelles (100 min, 105 mg polymer per $\mathrm{mL}$ citrate buffer $20 \mathrm{mM}$ ); (iv) tacrolimus loaded micelles (10 min penetration time); (v) tacrolimus loaded micelles (100 min penetration time); and (vi) tacrolimus loaded micelles (1000 min penetration time). Each skin sample had a size of $0.8 \mathrm{~cm} \times 0.8 \mathrm{~cm}$, then $10 \mu \mathrm{L}$ of the drug formulations was applied to the skin surface covering an area of ca. $0.4 \mathrm{~cm}$ $\times 0.4 \mathrm{~cm}$. After treatment, the samples were cut into $1 \mathrm{~mm} \times 2 \mathrm{~mm}$ pieces and were fixed in $2.5 \%$ glutaraldehyde (30 min at room temperature and overnight at $4{ }^{\circ} \mathrm{C}$ ). Subsequently, the samples were embedded using the standard protocol also applied in previous works $[11,12$, 15]. Briefly, the samples were post-fixed by $1 \% \quad \mathrm{OsO}_{4}$ and $0.8 \% \quad \mathrm{~K}_{4}\left[\mathrm{Fe}(\mathrm{CN})_{6}\right]$ in $0.1 \mathrm{M}$ cacodylate buffer. Dehydration of the samples was done by a sequence of washing steps in water-ethanol mixtures, finally using neat ethanol. Subsequently, the samples were embedded in EPON resin (Serva, Heidelberg, Germany). An ultramicrotome (Ultracut E, Leica, Germany) was used for preparing ca. $350 \mathrm{~nm}$ thick skin slices by cutting the skin samples vertically to the surface. The fixed skin slices were prepared in duplicates and were finally deposited on silicon nitride windows (thickness $100 \mathrm{~nm}$, Silson, Northampton, U.K.). All samples were characterized by optical microscopy prior to X-ray microscopy studies (Leica DM4000M, equipped with a Heidenhain linear encoder).

The experiments involving X-ray microscopy were performed at the Swiss Light Source at the PolLux instrument, which is located at a bending magnet providing linearly polarized soft $\mathrm{X}$-rays between $270 \mathrm{eV}$ and $1600 \mathrm{eV}$ [27-29]. The samples were mounted vertically on a 
high-precision piezoelectric stage and scanned horizontally. A Fresnel zone plate was used as focusing optics to generate high spatial resolution $(\sim 40 \mathrm{~nm})$ by geometrical demagnification of the exit slit. The transmitted signal was collected by using a small-area detector, consisting of a phosphor screen, which was coupled to a photomultiplier tube (Hamamatsu 647P). The instrument was operated under vacuum $\left(10^{-3} \mathrm{mbar}\right)$ for minimizing $X$-ray absorption by residual gas and to avoid sample contamination. Images were recorded at selected photon energies below and in the C 1s regime (270-320 eV) as well as below and in the $\mathrm{O} 1 \mathrm{~s}$ regime $(500 \mathrm{eV}$ and $550 \mathrm{eV})$. Image processing was carried out using IgorPro software similar to previous work $[12,13,15]$ as well as aXis2000 [30], and Origin ${ }^{\circledR}$ 2018b. 


\section{Results and Discussion}

Figure 1(a) shows the absorption cross section of the species under study in the $C$ 1s regime. This is required for gaining the chemical selectivity by $X$-ray microscopy. These spectra have not been reported before, so that they are briefly discussed. Note that the absorption spectra were left on a relative scale, but these can be easily converted into an absolute cross section scale by using the atomic absorption cross section, if the composition of the species under study is known, i.e. tacrolimus $\left(\mathrm{C}_{44} \mathrm{H}_{69} \mathrm{NO}_{12}, \mathrm{~m}=804.02 \mathrm{~g} / \mathrm{mol}\right)$ and the polymer composing the micelles $\left(\mathrm{C}_{289} \mathrm{H}_{454} \mathrm{~N}_{2} \mathrm{O}_{94}, \mathrm{M}=5454 \mathrm{~g} / \mathrm{mol}\right.$, cf. [22]), e.g. at $310 \mathrm{eV}$, i.e. a region, in which no resonant transitions occur. This yields for tacrolimus at $310 \mathrm{eV}$ a value of $38.9 \pm 0.5 \mathrm{Mbarn}$ (cf. Figure $1(\mathrm{a})$ ), if the pre-edge value at $280 \mathrm{eV}$ is set to $0 \mathrm{Mbarn}$. For the

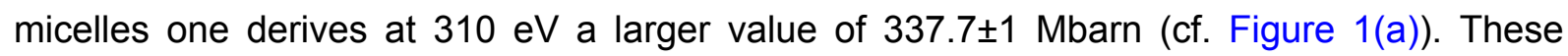
substantial cross sections, corresponding at the $C 1 \mathrm{~s} \rightarrow \pi^{*}$ transition of the micelles to 25 Mbarn and for tacrolimus to 16 Mbarn, are advantageous for the detection of topically applied substances, since these are roughly by a factor of 2.5 to 4 higher than at the 01 s-edge, as reported in earlier studies $[11,12]$.
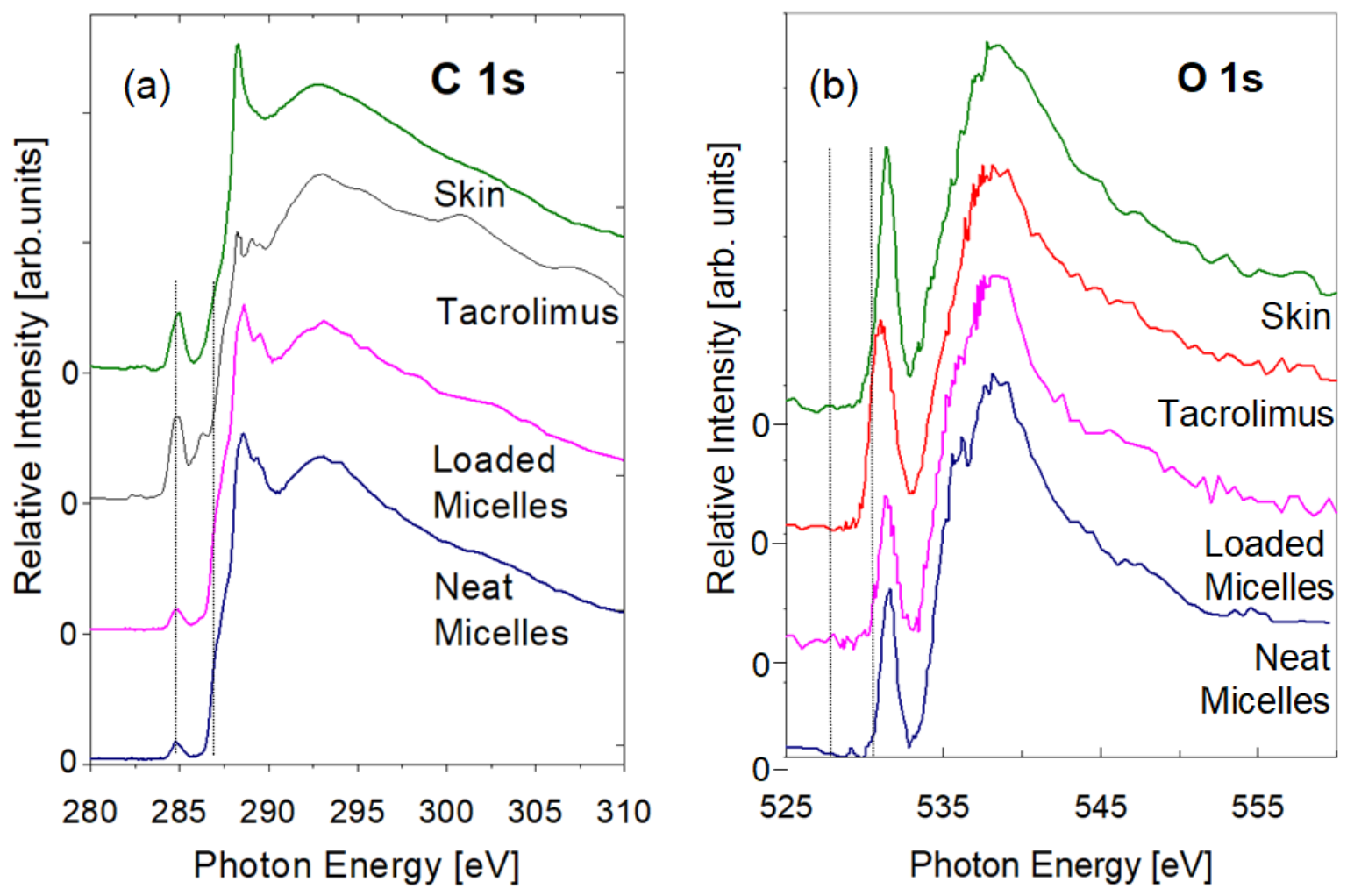

Figure 1: (a) Carbon 1s absorption cross section of fixed murine inflamed skin; tacrolimus; tacrolimus loaded micelles; and neat micelles. The vertical dashed lines at $284.6 \mathrm{eV}$ and $286.7 \mathrm{eV}$ correspond to the excitation energies used for X-ray microscopy studies; (b) Oxygen 1s absorption cross section of fixed murine inflamed skin; tacrolimus; tacrolimus loaded micelles; and neat micelles. The vertical dashed lines at $528.0 \mathrm{eV}$ and $530.3 \mathrm{eV}$ correspond to the excitation energies used for X-ray microscopy studies. 
This is a clear advantage of the instrument at the Swiss Light Source which barely shows any contamination of the X-ray microscope and the beamline in the $\mathrm{C} 1 \mathrm{~s}$ regime (cf. ref. [31]), specifically if compared to other X-ray microscopy installations. The chemical composition of fixed skin is not exactly known, so that the conversion into an absolute vertical scale may lead to substantial errors (cf. Figure 1(a)). For the tacrolimus-loaded micelles (see Figure 1(a)) one expects a similar $\mathrm{C}$ 1s-spectrum compared to neat micelles, since the drug loading is estimated to be of the order of $0.7 \%$ (see above). As a result, tacrolimus will not significantly affect the spectral shape of the tacrolimus-loaded micelles.

The assignment of the spectral features shown in Figure 1(a) is straightforward according to the molecular structure of the absorbing carbon in all species under study [32, 33]. Note that detailed assignments require quantum chemical calculations (cf. Ref. [11]), which go beyond the scope of this work. The distinct resonance located for tacrolimus at $284.58 \mathrm{eV}$ is due to the $\mathrm{C} 1 \mathrm{~s} \rightarrow \pi^{*}$ resonance of the carbonyl groups, of which there are four in tacrolimus (see Scheme 1(a)). The distinct features near $290 \mathrm{eV}$ are likely due to $\sigma^{*}(\mathrm{C}-\mathrm{H})$ excitations [33]. At slightly higher energy, i.e. near $293 \mathrm{eV}$, one expects the $\sigma^{*}(\mathrm{C}-\mathrm{O})$ - and $\sigma^{*}(\mathrm{C}-\mathrm{C})$-excitations, whereas the $\sigma^{*}(\mathrm{C}-\mathrm{C})$-excitations occur as broad and weak features above $300 \mathrm{eV}$. The $\mathrm{C} 1 \mathrm{~s}$ absorption spectrum of the micelles is similar in shape, but there are small differences in absorption energies, such as that the $\mathrm{C} \rightarrow \pi^{*}$-resonance peaks at slightly higher energy than for tacrolimus, i.e. at $285.0 \mathrm{eV}$. Note that there is no shift in absorption energy compared to fixed murine skin (see Figure 1(a)), so that we cannot use the same approach that was successfully applied for probing dexamethasone in fixed human skin, where a distinct spectral shift of the $\mathrm{O} 1 \mathrm{~s} \rightarrow \pi^{*}$ resonances of skin and the drug allowed us to selectively probe the drug $[11,12]$. However, in the present case it appears to be possible to use two photon energies at which there is a change in differential absorption primarily for the micelles, but not for fixed skin. These photon energies are found at $284.6 \mathrm{eV}$ and $286.7 \mathrm{eV}$, respectively. There, the micelles gain absorption cross section by $350 \pm 5 \%$, whereas for tacrolimus a drop in cross section $23 \pm 5 \%$ is observed. This implies that changes in absorption cross section due to topically applied micelles can be selectively probed in the $\mathrm{C} 1 \mathrm{~s}$-regime, but not for tacrolimus.

The $\mathrm{O} 1 \mathrm{~s}$ absorption regime is shown in Figure $1(\mathrm{~b})$ for the same samples discussed for the C 1s regime (cf. Figure 1(a)). The assignments are similar to those given above for the $C$ 1s features with the difference that only oxygen sites are excited. The intense resonances peaking near $531 \mathrm{eV}$ are due to the $\mathrm{O} 1 \mathrm{~s} \rightarrow \pi^{*}$ transition. The broad feature near $540 \mathrm{eV}$ is assigned to the $O 1 \mathrm{~s} \rightarrow \sigma^{*}$ transition. Note that the $O$ 1s absorption cross section is 
significantly smaller than that at the C 1s-edge, which is only used in this work for supplementary studies, such as probing tacrolimus.

Figure 2 shows a series of X-ray micrographs from the top skin layers of inflamed murine skin. These were recorded in the $C$ 1s regime in order to detect the presence and penetration of polymeric micelles after exposure of the skin samples to topically applied substances for $100 \mathrm{~min}$. Figure 2(a)-(d) shows a series of images taken at $286.7 \mathrm{eV}$. This photon energy corresponds to the onset of the $C$ 1s continuum (cf. Figure 1(a)) for all topically applied substances as well as fixed murine skin.
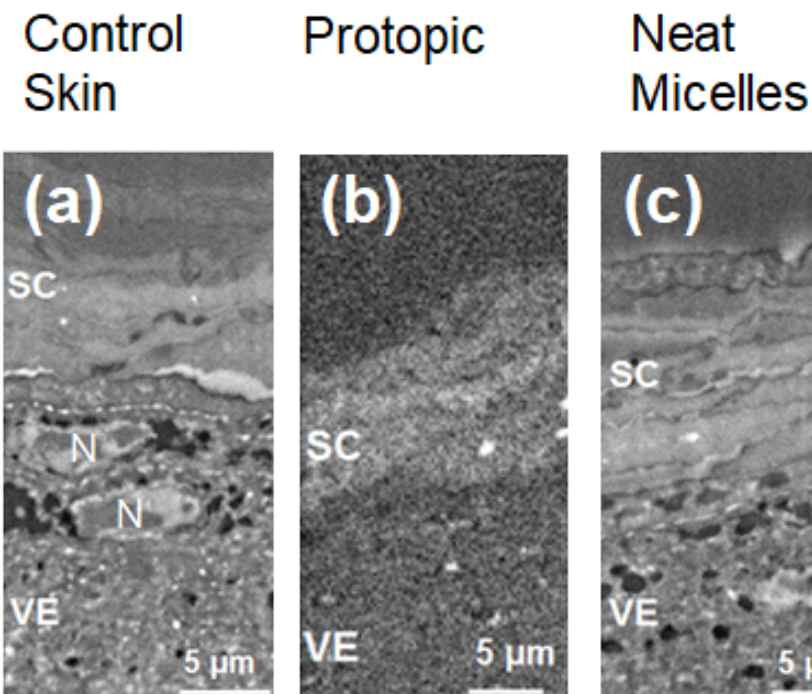

\section{Loaded}
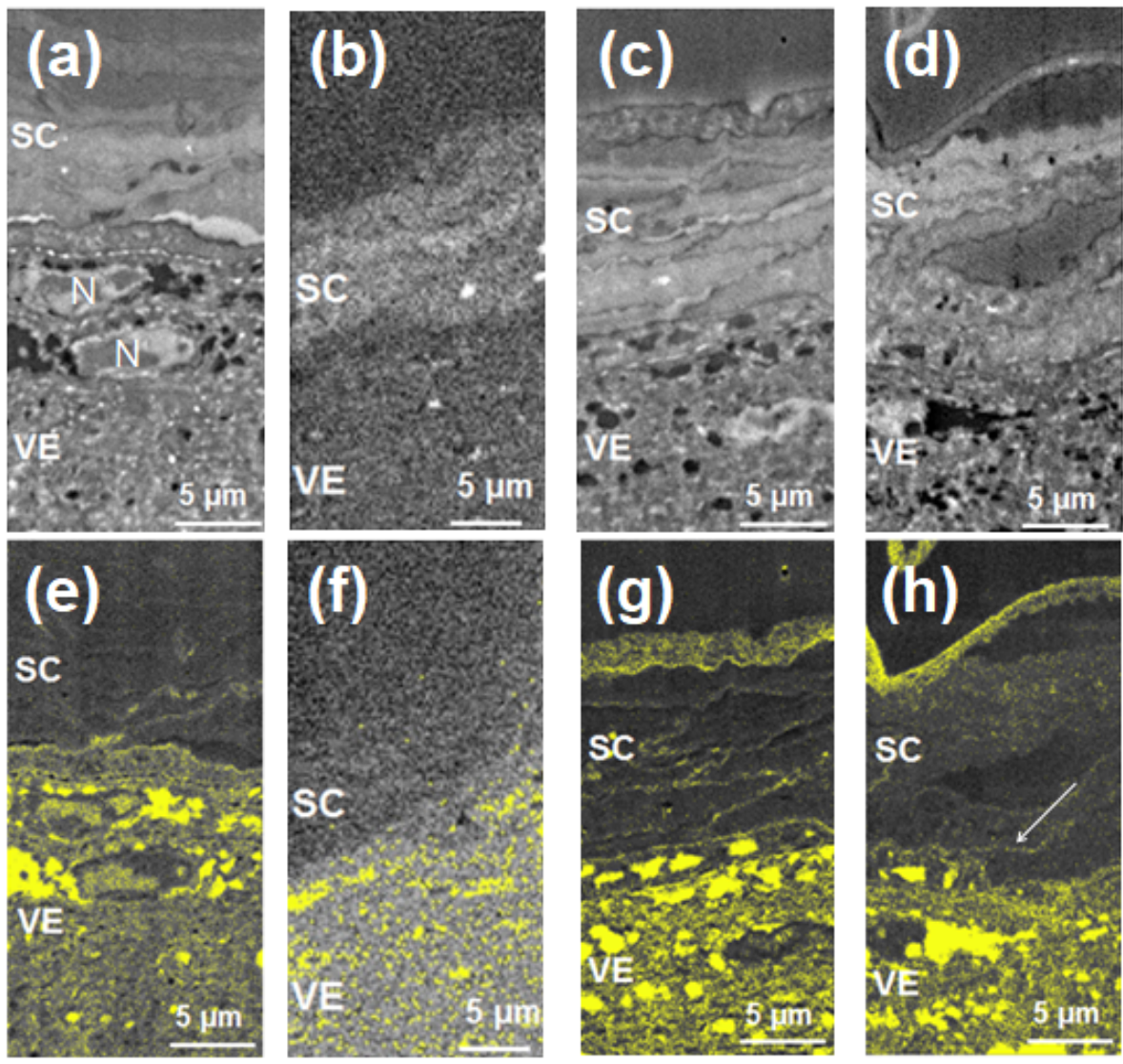

Figure 2: X-ray micrographs of murine inflamed skin near the skin surface: SC: stratum corneum, VE: viable epidermis, N: nucleus. The micrographs (a) - (d) have been taken at $286.7 \mathrm{eV}$ for determining the structure of the samples: (a) control skin; (b) skin treated with Protopic ${ }^{\circledR}$; (c) skin treated with neat micelles; (d) skin treated with tacrolimus loaded micelles. The micrographs shown in the lower row (e) - (h) are derived from changes in optical density between $284.6 \mathrm{eV}$ and $286.7 \mathrm{eV}$ for probing efficiently micelles. Changes in optical density above a common threshold value are indicated by yellow color, similar to previous work [15]. The arrow in (h) points toward enhanced concentration of micelles at the border to the viable epidermis. Note that similar features also occur in (e) and $(\mathrm{g})$. The scale bars correspond to 5 $\mu \mathrm{m}$. Note that all micrographs were taken with $50 \mathrm{~nm}$ step widths, except for (b) and (f), where $200 \mathrm{~nm}$ were chosen. See text for further details. 
Each micrograph shows the stratum corneum with distinct layers of corneocytes besides the top part of the viable epidermis. In contrast, the images in Figure 2(e)-(h) indicate the presence of micelles at detectable levels. Specifically, Figure 2(a)-(d) shows the structural properties of the skin samples. The distinct layers in the stratum corneum have typically a thickness of $1.6 \mu \mathrm{m}$ and appear to be swollen due to the topical application of $\mathrm{NaCl}$ solution (Figure 2(a)), Protopic $^{\circledR}$ (Figure 2(b), neat micelles (Figure (2(c)), and tacrolimus loaded micelles (Figure (2d)). Below these structures lies the viable epidermis, showing sections of keratinocytes with nuclei (partially indicated by $N$, e.g. in Figure 2(a) - see also Figure 2(e)(h)) and provides the chemical contrast due to selective excitation at $284.6 \mathrm{eV}$ and $286.7 \mathrm{eV}$, respectively. These energies were chosen in order to have the same absorption contrast for fixed skin, while for the other samples, i.e. micelles and tacrolimus-loaded micelles, an increase in absorption cross section leads to an enhancement of the differential absorption. We have chosen a presentation for these Figures that is similar to previous work (cf. ref. [15]) in order to visualize the topically applied species under study. This means that a common threshold value is used at which the gray scale image turns into yellow color of increased differential absorption. This shows a constant background in the viable epidermis for all micrographs (see Figure 2(e)-(h)), which is not considered for the present analysis. We assume that these highlighted structures are due to carbon-rich moieties in the upper part of the viable epidermis, which can be assigned to granular and lamellar patterns according to electron microscopy studies $[34,35]$. Note that these occur only in the stratum granulosum and below, but not in the stratum corneum, yielding a chemical contrast, which is similar to the topically applied substances under study. Caprylic acid, which is a chemical building block of the micelles' polymer, is a fatty acid. High prevalence of lipids, which are endogenously present in the skin, might be the reason for signal interference in the viable epidermis. For example, lipid species that have been detected particularly in the epidermis, but not in the stratum corneum, are phosphatidylcholins [36].

In Figure 2(a) and (e) it is also evident that cell nuclei in the stratum granulosum, indicated by $\mathrm{N}$, are barely affected by this cross sensitivity. Despite this cross sensitivity in the viable epidermis, it is possible to probe topically applied drug formulations in the stratum corneum. This means that the stratum corneum of the reference skin sample does not show any enhanced absorption contrast in the top of this skin layer. Furthermore, Protopic ${ }^{\circledR}$ (cf. Figure 2(b) and (f)) does not show any hint for tacrolimus penetration, implying that only small quantities can penetrate, which are evidently below the detection limit, if probed in the $C 1 \mathrm{~s}$ regime. However, the skin samples exposed to micelles display much higher signals in the stratum corneum. As noted above, the sensitivity is significantly higher for probing micelles as compared to tacrolimus. Neat micelles show evidence of their penetration most intensely 
in a narrow range at the top of the stratum corneum (see Figure 2(c) and (g)). Here, the entire corneocytes in the top layer appear to have taken up the formulation. The corneocytes show a thickness of $1.8 \pm 0.2 \mu \mathrm{m}$, which is thicker than in untreated murine skin [37]. Thus, it is assumed that the corneocytes are swollen due to topical treatment. In the lower layers of the stratum corneum one observes, as indicated by yellow color, the micelles preferably at the edges of corneocytes. This hints that their penetration is mostly due to transport in the lipophilic lamellae [38], as is known to occur for hydrophobic low molecular weight drugs [12]. Swelling of corneocytes is known for topical application of polymeric nanogel formulations, e.g. from thermoresponsive nanogels [39]. When exposed to micelles loaded with tacrolimus, the following changes are observed: The top layer of the stratum corneum contains also most of the topically applied species, but the load in lower parts of the stratum corneum shows a different signature compared to the neat micelles. In the case of the drug-loaded micelles one observes a more delocalized signal distribution with lower relative concentration (see Figure 2(d) and (h)). Localized structures at the edges of corneocytes only occur in deeper layers of the stratum corneum, as indicated by the white arrow in Figure 2(h). The delocalized signal in the stratum corneum shown in Figure 2(h) may indicate the disintegration of some micelles in superficial skin layers and their ability to diffuse down and accumulate in the stratum granulosum. At the same time, the micelles might be able to carry with them the drug into layers below the superficial stratum corneum, as previously reported [22].

Figure 3 summarizes the findings after $100 \mathrm{~min}$ penetration time as follows: Integrated intensities of the different layers, i.e. stratum corneum (SC) and viable epidermis (VE) are added, yielding an averaged response due to changes in differential absorption between $286.7 \mathrm{eV}$ and $284.6 \mathrm{eV}$, respectively, where the differential absorption of fixed murine skin cancels out. The control skin and the Protopic ${ }^{\circledR}$ treated samples show in the stratum corneum a minor response in terms of differential absorption. In contrast, the response is significantly higher for neat micelles and tacrolimus loaded micelles, indicating the feasibility to show the presence of micelles in the stratum corneum. In contrast, differential absorption is almost the same for control and viable epidermis treated with Protopic $^{\circledR}$, neat micelles, and tacrolimus loaded micelles. This implies that penetration of micelles into the viable epidermis cannot be evidenced by this technique. However, it is assumed in accordance with previous studies that they will not penetrate into this skin layer [15]. Additionally, the role of tacrolimus cannot be fully derived from these experiments, since the drug shows at the chosen photon energies even a drop in differential absorption, as displayed in Figure 1(a). 


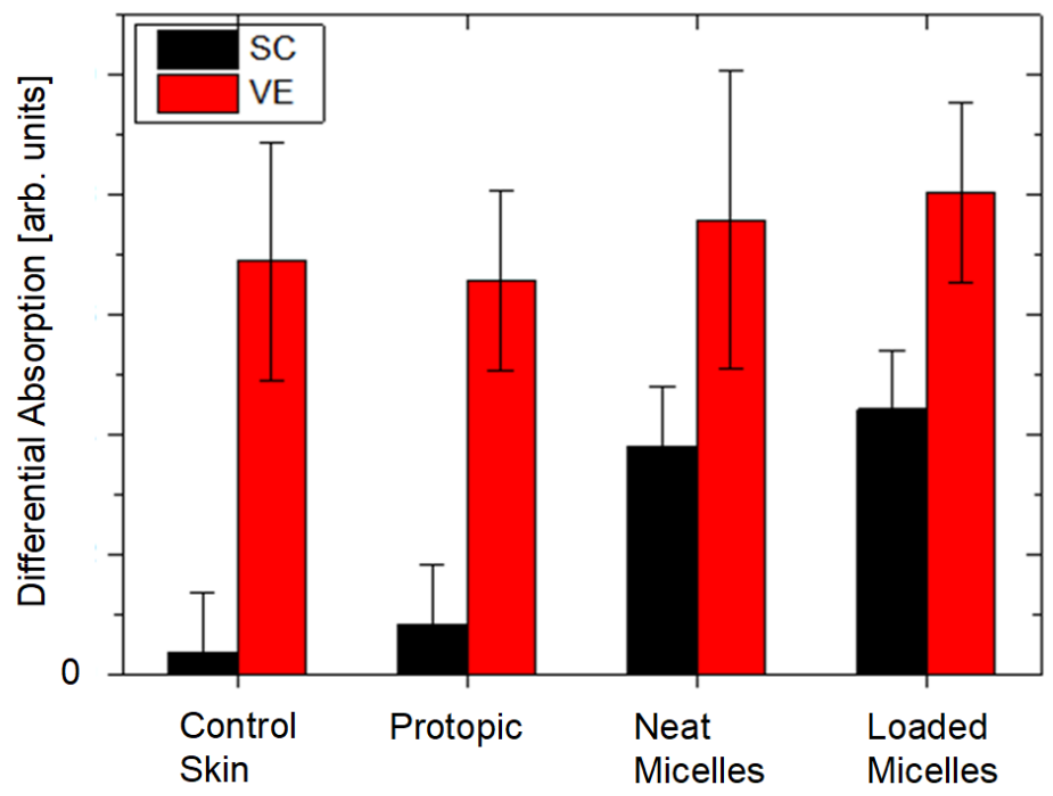

Figure 3: Changes in differential absorption for probing micelles by X-ray microscopy in different skin layers and differently treated skin samples for 100 min as indicated. The error bars correspond to the variance of the corresponding signal levels, where a quarter of the respective region was evaluated. Stratum corneum (SC) and viable epidermis (VE) - see text for further details.

In comparison to previous work on polymeric micellar nanocarriers transporting tacrolimus these results are fully compatible [22]. Core-multishell nanocarriers were found to remain in the stratum corneum and there was no or minimal penetration into deeper layers observed. This was also the case for topically applied thermoresponsive nanogels [39], indicating the necessity of small sized nanocarriers and specific physicochemical properties in order to cross the skin barriers. As a result, this type of drug delivery is ideally expected to minimize the systemic exposure to the drug.

Figure 4 shows the temporal skin penetration behavior in samples treated with loaded micelles, as probed by an increase in differential absorption in the stratum corneum. We have taken $\mathrm{X}$-ray micrographs for three penetration times, i.e. at $10 \mathrm{~min}, 100 \mathrm{~min}$, and 1000 $\min$. 


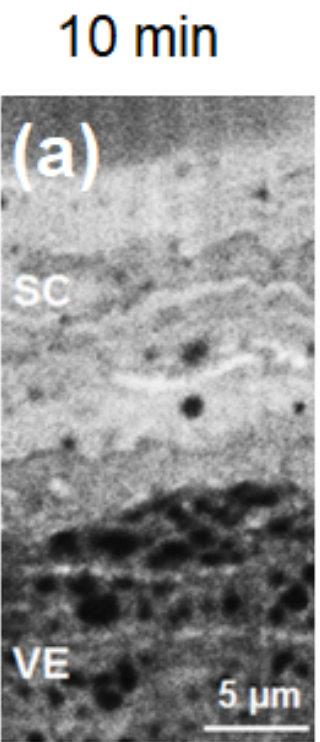

\section{$100 \mathrm{~min}$}
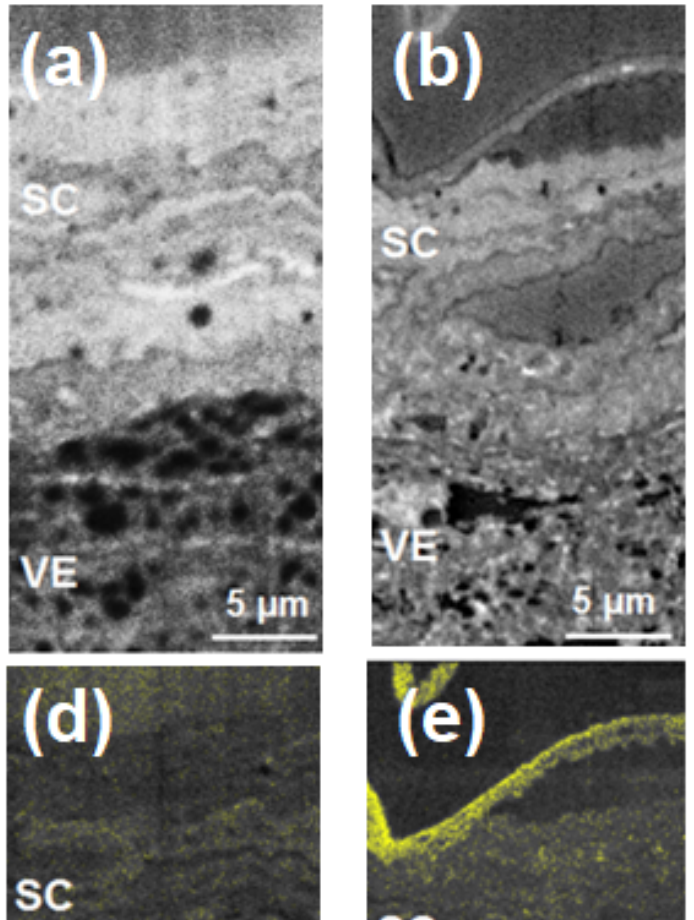

SC

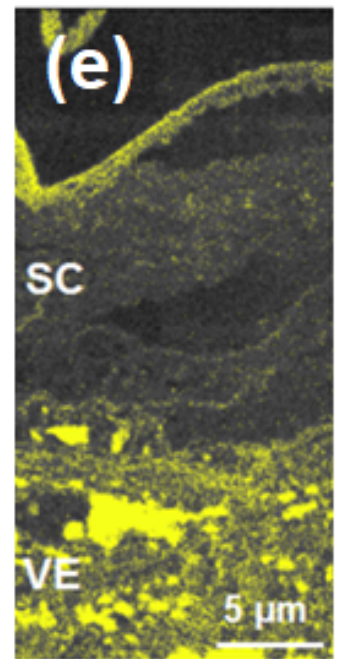

\section{$1000 \mathrm{~min}$}
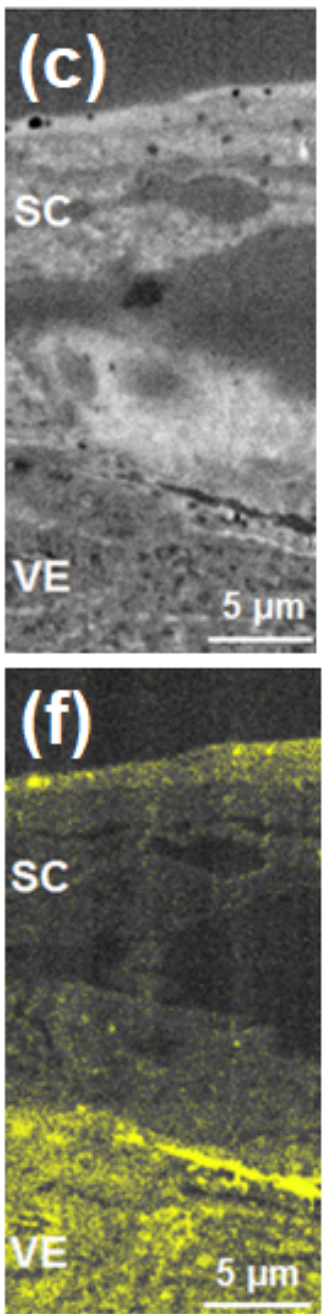

Figure 4: X-ray micrographs of inflamed murine skin near the skin surface: SC: stratum corneum, VE: viable epidermis. The micrographs (a) - (c) have been taken at $286.7 \mathrm{eV}$ for determining the structure of the samples: (a) skin treated for 10 min. penetration time with tacrolimus loaded micelles; (b) 100 min penetration time; (c) 1000 min penetration time. The micrographs shown in the lower row (d) - (f) are derived from changes in optical density, i.e. at $284.6 \mathrm{eV}$ and $286.7 \mathrm{eV}$, probing mostly micelles. Changes in optical density above a common threshold value are indicated by yellow color. Note that all micrographs were taken with $50 \mathrm{~nm}$ step widths. See text for further details.

The presence of micelles was evidenced in the stratum corneum at $100 \mathrm{~min}$ and 1000 minutes. Clearly, only a minor signal from the micelles is detected in the stratum corneum after 10 min penetration time. This is indicated by light yellow color in Figure 4(d). Most of the intensity is observed even above the stratum corneum in the top part of this image (not shown in Figure 4(d)). The measurements taken at $100 \mathrm{~min}$ are identical to those shown in Figure 2(c) and (g). As the penetration time is increased to $100 \mathrm{~min}$, an intense signal is observed in the entire top layer of corneocytes, implying that this layer has absorbed the drug formulation (cf. Figure 4(e)). This situation is similar, but more delocalized after 1000 
min. penetration time, as displayed in Figure 4(f). This might indicate the disintegration of the micelles and release of tacrolimus into the tissue. A quantification of these results cannot be done from the micrographs, which is due to the low signal strength. Therefore, the relative intensities of the micelles in the stratum corneum have been derived from the results shown in Figure 4, where cross sensitivities in the viable epidermis impede analysis below the stratum corneum. The results are shown in Figure 5. The differential absorption due to micelles increases in the stratum corneum (black bars) with time, whereas the intensity in the viable epidermis (red bars) remains almost constant. This leads to the conclusion that the temporally resolved loading of the stratum corneum appears to go into saturation for penetration times above $100 \mathrm{~min}$. Such penetration behavior will ensure safety due to a ratelimited drug uptake by the skin.

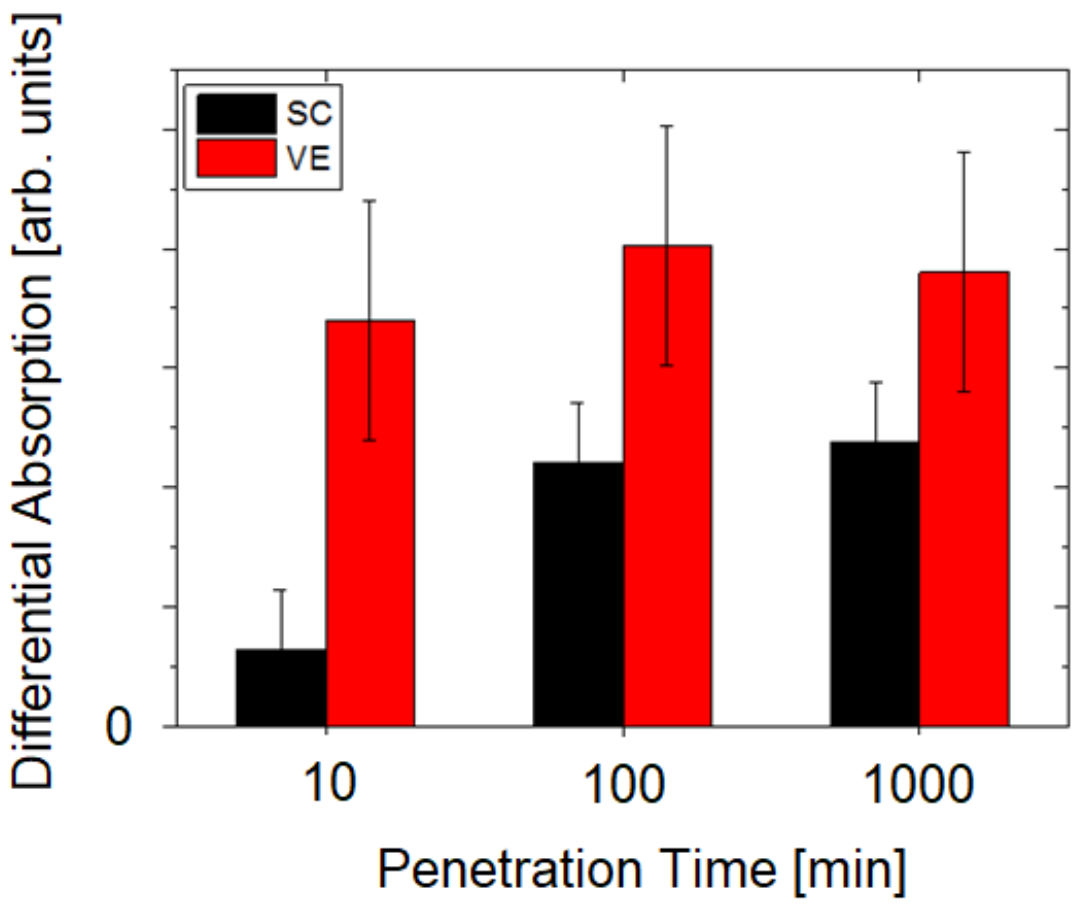

Figure 5: Changes in differential absorption in the top skin layers of tacrolimus loaded micelles as a function of penetration time. The error bars correspond to the variance of the corresponding signal levels, where a quarter of the respective region was evaluated. Stratum corneum (SC) and viable epidermis (VE) - see text for further details.

A limitation of these results is that one cannot distinguish between neat micelles, tacrolimus loaded micelles, and tacrolimus on the X-ray micrographs shown in Figures 2 and 4 with the C 1s probing technique. As pointed out above, there is an increase in absorption for the micelles, providing the chemical contrast in the X-ray micrographs, whereas for tacrolimus there is even a slight drop in absorption, if the photon energies at $284.6 \mathrm{eV}$ and $286.7 \mathrm{eV}$ are used. This is, however, not suitable for detecting tacrolimus via resonant excitation in the $C$ 

tacrolimus shows an $\mathrm{O} 1 \mathrm{~s} \rightarrow \pi^{*}$ resonance, which is positioned at slightly lower energy than the other species under study. This allows us to selectively probe the drug in fixed murine skin. The differential absorption is adjusted at $528.0 \mathrm{eV}$ (pre-edge regime) and at $530.3 \mathrm{eV}$ (slope of the $01 \mathrm{~s} \rightarrow \pi^{*}$ transition (cf. vertical lines in Figure 1(b)). There, the cross sensitivity is as small as possible, while the absorption cross section is appreciably high, corresponding to $12.0 \pm 0.5 \mathrm{Mbarn}$, if the atomic absorption cross section in the $\mathrm{O} 1 \mathrm{~s}$-continuum is used (see above). Then, one can derive for the samples under study the differential absorption, which also shows a background due to cross sensitivities of the majority species in the skin samples. Figure 6 clearly indicates that there is a distinct difference in differential absorption in the stratum corneum, providing evidence that tacrolimus is probed with weak intensity. A lower level of differential absorption is obtained from the control sample and the skin sample containing neat micelles (see Figure 6(a)), whereas an enhanced level is obtained from the Protopic $^{\circledR}$ sample, which is a similar, but even slightly higher, for the tacrolimus loaded micelles. This underscores the previously noticed favorable penetration of tacrolimus, if formulated in micelles rather than in Protopic ${ }^{\circledR}[21,22]$. We note that the drug load is lower in the viable epidermis, so that this analysis is not extended to this skin region. We have rather evaluated the time-resolved measurements, as shown in Figure 6(b).

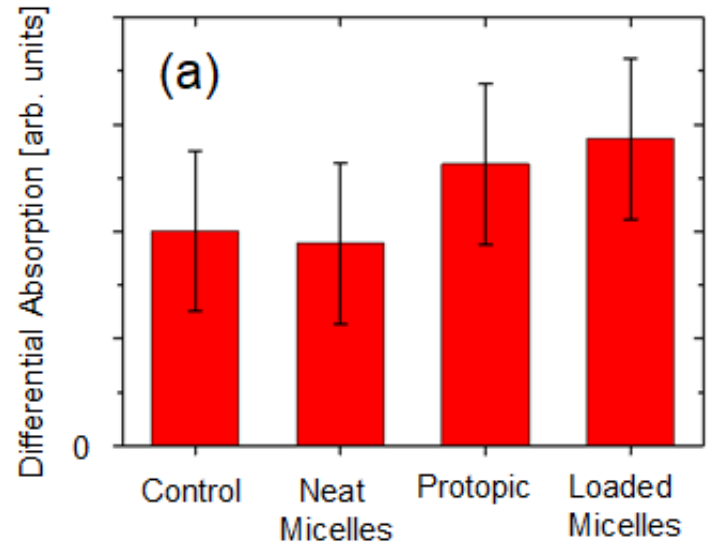

Figure 6: Changes in differential absorption in the stratum corneum due to tacrolimus probed at the $\mathrm{O}$ 1s-edge relative to a untreated control sample (cf. Figure 1(b)): (a) Changes in differential absorption for samples exposed for $100 \mathrm{~min}$ to neat micelles, Protopic ${ }^{\circledR}$, and tacrolimus loaded micelles; (b) temporal evolution of the tacrolimus load in the stratum corneum after delivery with tacrolimus loaded micelles - see text for further details. The error bars correspond to the variance of the corresponding signal levels, where a quarter of the respective region was evaluated.

The $\mathrm{O}$ 1s probing technique allowed for following tacrolimus levels in the stratum corneum as a function of penetration time. The same level of differential absorption is observed for the control sample and $10 \mathrm{~min}$ penetration time. This means that tacrolimus has not yet 
penetrated into the stratum corneum. There is an increase of tacrolimus levels at $100 \mathrm{~min}$ penetration time, with slightly decreasing levels at $1000 \mathrm{~min}$. The local concentration of tacrolimus might have dropped in superficial layers due to transport into deeper skin layers, but this remains speculative at this point. Due to rate-limited drug uptake by the skin, drug concentrations might remain at safe, but active levels in a considered volume.

\section{Summary and Conclusions}

X-ray microscopy has been applied for the first time to follow the penetration of tacrolimus in inflamed murine skin ex vivo. Element selective excitation allowed us to probe the penetration of the drug and micelles in the top skin layers, where C 1s-excitation is preferable for probing the micelles and $\mathrm{O}$ 1s-excitation is more suitable for probing tacrolimus. The local concentration of the drug and micelles is weak in the stratum corneum, but improved compared to Protopic $^{\circledR}$, a commercial formulation. The present results indicate that the corneocytes are swollen and penetrated by the drug formulation, but there is also minor evidence for enhanced concentration near the lipid lamellae between the corneocytes. Additional time-resolved studies indicate that the local concentration of micelles and tacrolimus in the stratum corneum is increasing with time and reaching finally saturation.

\section{Acknowledgments}

We gratefully acknowledge financial support by DFG within SFB 1112 (projects B02, C03, C04) and project RU420/12-1. The PolLux end station was financed by the German Minister für Bildung und Forschung (BMBF) through contracts 05KS4WE1/6 and 05KS7WE1. 


\section{References}

[1] A. Gysler, B. Kleuser, W. Sippl, K. Lange, H.C. Korting, H.D. Höltje, M. Schäfer-Korting, Skin penetration and metabolism of topical glucocorticoids in reconstructed epidermis and in excised human skin, Pharm. Res., 16 (1999) 1386-1391.

[2] D. Selzer, M.M.A. Abdel-Mottaleb, T. Hahn, U.F. Schaefer, D. Neumann, Finite and infinite dosing: Difficulties in measurements, evaluations and predictions, Adv. Drug. Del. Rev., 65 (2013) 278-294.

[3] J.D. Bos, M.M.H.M. Meinardi, The 500 Dalton rule for the skin penetration of chemical compounds and drugs, Exp. Dermatol., 9 (2000) 165-169.

[4] Y.K. Lin, S.H. Yang, C.C. Chen, H.C. Kao, J.Y. Fang, Using Imiquimod-Induced Psoriasis-Like Skin as a Model to Measure the Skin Penetration of Anti-Psoriatic Drugs, Plos One, 10 (2015) e0137890.

[5] E. Rühl, Probing Functionalized Nanoparticles in Biological Media, in: K. Wandelt (Ed.) Encyclopedia of Interfacial Chemistry, Elsevier, Oxford, 2018, pp. 795-802.

[6] C.W. Freudiger, W. Min, B.G. Saar, S. Lu, G.R. Holtom, C. He, J.C. Tsai, J.X. Kang, X.S. Xie, Label-Free Biomedical Imaging with High Sensitivity by Stimulated Raman Scattering Microscopy, Science, 322 (2008) 1857-1861.

[7] Q. Li, T. Zhang, Y. Pan, L.C. Ciacchi, B. Xu, G. Wei, AFM-based force spectroscopy for bioimaging and biosensing, RSC Adv., 6 (2016) 12893-12912.

[8] P. Verma, Tip-Enhanced Raman Spectroscopy: Technique and Recent Advances, Chem. Rev., 117 (2017) 6447-6466.

[9] A. Danielli, K.I. Maslov, A. Garcia-Uribe, A.M. Winkler, C. Li, L. Wang, Y. Chen, G.W. Dorn, L.V. Wang, Label-free photoacoustic nanoscopy, J. Biomed. Opt., 19 (2014) 086006.

[10] A. Dazzi, C.B. Prater, AFM-IR: Technology and Applications in Nanoscale Infrared Spectroscopy and Chemical Imaging, Chem. Rev., 117 (2017) 5146-5173.

[11] K. Yamamoto, R. Flesch, T. Ohigashi, S. Hedtrich, A. Klossek, P. Patoka, G. Ulrich, S. Ahlberg, F. Rancan, A. Vogt, U. Blume-Peytavi, P. Schrade, S. Bachmann, M. SchäferKorting, N. Kosugi, E. Rühl, Selective probing of the penetration of dexamethasone into human skin by soft X-ray spectromicroscopy, Anal. Chem., 87 (2015) 6173-6179.

[12] K. Yamamoto, A. Klossek, A. Flesch, F. Rancan, M. Weigand, I. Bykova, M. Bechtel, S. Ahlberg, A. Vogt, U. Blume-Peytavi, P. Schrade, S. Bachmann, S. Hedtrich, M. Schäfer-Korting, E. Rühl, Influence of the skin barrier on the penetration of topicallyapplied dexamethasone probed by soft X-ray spectromicroscopy, Eur. J. Pharm. Biopharm., 118 (2017) 30-37.

[13] R. Schulz, K. Yamamoto, A. Klossek, R. Flesch, S. Hönzke, F. Rancan, A. Vogt, U. Blume-Peytavi, S. Hedtrich, M. Schäfer-Korting, E. Rühl, R.R. Netz, Data-based 
modeling of drug penetration relates human skin barrier function to the interplay of diffusivity and free-energy profiles, Proc. Nat. Acad. Sci. U.S., 114 (2017) 3631-3636.

[14] J.M. Brandner, Importance of tight junctions in relation to skin barrier function, Curr. Probl. Dermatol., 49 (2016) 27-37.

[15] K. Yamamoto, A. Klossek, R. Flesch, T. Ohigashi, E. Fleige, F. Rancan, J. Frombach, A. Vogt, U. Blume-Peytavi, P. Schrade, S. Bachmann, R. Haag, S. Hedtrich, M. Schäfer-Korting, N. Kosugi, E. Rühl, Core-multishell nanocarriers: Transport and release of dexamethasone probed by soft X-ray spectromicroscopy, J. Control. Release, 242 (2016) 64-70.

[16] U. Alexiev, P. Volz, A. Boreham, R. Brodwolf, Time-resolved fluorescence microscopy (FLIM) as an analytical tool in skin nanomedicine, Eur. J. Pharm. Biopharm., 116 (2017) 111-124.

[17] H. Pischon, M. Radbruch, A. Ostrowski, P. Volz, C. Gerecke, M. Unbehauen, S. Hönzke, S. Hedtrich, J.W. Fluhr, R. Haag, B. Kleuser, U. Alexiev, A.D. Gruber, L. Mundhenk, Stratum corneum targeting by dendritic core-multishell-nanocarriers in a mouse model of psoriasis, Nanomed. Nanotechnol., 13 (2017) 317-327.

[18] M. Radbruch, H. Pischon, A. Ostrowski, P. Volz, R. Brodwolf, F. Neumann, M. Unbehauen, B. Kleuser, R. Haag, N. Ma, U. Alexiev, L. Mundhenk, A.D. Gruber, Dendritic Core-Multishell Nanocarriers in Murine Models of Healthy and Atopic Skin, Nanoscale Res. Lett., 12 (2017) 64.

[19] T. Ruzicka, T. Bieber, E. Schopf, A. Rubins, A. Dobozy, J.D. Bos, S. Jablonska, I. Ahmed, K. ThestrupPedersen, F. Daniel, A. Finzi, S. Reitamo, A short-term trial of tacrolimus ointment for atopic dermatitis, New Engl. J. Med., 337 (1997) 816-821.

[20] A. Menter, N.J. Korman, C.A. Elmets, S.R. Feldman, J.M. Gelfand, K.B. Gordon, A. Gottlieb, J.Y.M. Koo, M. Lebwohl, H.W. Lim, A.S. Van Voorhees, K.R. Beutner, R. Bhushan, Guidelines of care for the management of psoriasis and psoriatic arthritis Section 3. Guidelines of care for the management and treatment of psoriasis with topical therapies, J. Am. Acad. Dermatol., 60 (2009) 643-659.

[21] D. Gabriel, T. Mugnier, H. Courthion, K. Kranidioti, N. Karagianni, M.C. Denis, M. Lapteva, Y. Kalia, M. Moller, R. Gurny, Improved topical delivery of tacrolimus: A novel composite hydrogel formulation for the treatment of psoriasis, J. Control. Release, 242 (2016) 16-24.

[22] M. Lapteva, K. Mondon, M. Möller, R. Gurny, Y.N. Kalia, Polymeric micelle nanocarriers for the cutaneous delivery of tacrolimus: A targeted approach for the treatment of psoriasis, Mol. Pharm., 11 (2014) 2989-3001. 
[23] W. Lei, C. Yu, H. Lin, X. Zhou, Development of tacrolimus-loaded transfersomes for deeper skin penetration enhancement and therapeutic effect improvement in vivo, Asian J. Pharm. Sci., 8 (2013) 336-345.

[24] D. Dheer, Jyoti, P.N. Gupta, R. Shankar, Tacrolimus: An updated review on delivering strategies for multifarious diseases, Eur.J. Pharm. Sci., 114 (2018) 217-227.

[25] A.S.B. Goebel, R.H.H. Neubert, J. Wohlrab, Dermal targeting of tacrolimus using colloidal carrier systems, Int. J. Pharm., 404 (2011) 159-168.

[26] Drug Bank, in, https://www.drugbank.ca/drugs/DB00864, 2018.

[27] J. Raabe, G. Tzvetkov, U. Flechsig, M. Böge, A. Jaggi, B. Sarafimov, M.G.C. Vernooij, T. Huthwelker, H. Ade, D. Kilcoyne, T. Tyliszczak, R.H. Fink, C. Quitmann, PolLux: A new facility for soft $x$-ray spectromicroscopy at the Swiss Light Source, Rev. Sci. Instrum., 79 (2008) 113704.

[28] U. Flechsig, C. Quitmann, J. Raabe, M. Böge, R. Fink, H. Ade, The PolLux Microspectroscopy Beam line at the Swiss Light Source, AIP Conf. Proc., 879 (2007) 505-508.

[29] U. Frommherz, J. Raabe, B. Watts, R. Stefani, U. Ellenberger, Higher Order Suppressor (HOS) for the PolLux Microspectroscope Beamline at the Swiss Light Source SLS, AIP Conf. Proc., 1234 (2010) 429-432.

[30] A.P. Hitchock, aXis 2000 - Analysis of X-ray Images and Spectra, in: http://unicorn.mcmaster.ca/axis/aXis2000-windows-pre-IDL8.3.html, $\quad$ McMaster University, Hamilton, Ont. Canada, 2018.

[31] B. Watts, N. Pilet, B. Sarafimov, K. Witte, J. Raabe, Controlling optics contamination at the PolLux STXM, J. Instrum., 13 (2018) C04001.

[32] A.P. Hitchcock, D.C. Mancini, Bibliography and database of inner-shell excitationspectra of gas phase atoms and molecules, J. El. Spectrosc. Relat. Phenom., 67 (1994) 1-132.

[33] J. Stöhr, NEXAFS Sepctroscopy, Springer, Berlin, 1992.

[34] A. Al-Amoudi, J. Dubochet, L. Norlén, Nanostructure of the epidermal extracellular space as observed by cryo-electron microscopy of vitreous sections of human skin, J. Invest. Dermatol., 124 (2005) 764-777.

[35] A. Narangifard, L. den Hollander, C.L. Wennberg, M. Lundborg, E. Lindahl, I. Iwai, H. Han, S. Masich, B. Daneholt, L. Norlén, Human skin barrier formation takes place via a cubic to lamellar lipid phase transition as analyzed by cryo-electron microscopy and EM-simulation, Exp. Cell Res., 366 (2018) 139-151.

[36] C.A. Mitchell, H. Long, M. Donaldson, S. Francese, M.R. Clench, Lipid changes within the epidermis of living skin equivalents observed across a time-course by MALDI-MS imaging and profiling, Lipids Health Dis., 14 (2015) 84. 
[37] S.Y.E. Hou, A.K. Mitra, S.H. White, G.K. Menon, R. Ghadially, P.M. Elias, Membrane Structures in Normal and Essential Fatty Acid-Deficient Stratum Corneum: Characterization by Ruthenium Tetroxide Staining and X-Ray Diffraction, J. Invest. Dermatol., 96 (1991) 215-223.

[38] J.A. Bouwstra, P.L. Honeywell-Nguyen, G.S. Gooris, M. Ponec, Structure of the skin barrier and its modulation by vesicular formulations, Prog. Lipid Res., 42 (2003) 1-36.

[39] M. Giulbudagian, F. Rancan, A. Klossek, K. Yamamoto, J. Jurisch, V.C. Neto, P. Schrade, S. Bachmann, E. Rühl, U. Blume-Peytavi, A. Vogt, M. Calderon, Correlation between the chemical composition of thermoresponsive nanogels and their interaction with the skin barrier, J. Control. Release, 243 (2016) 323-332. 